\title{
Nano-multilamellar lipid vesicles (NMVs) enhance protective antibody responses against Shiga toxin (Stx2a) produced by enterohemorrhagic Escherichia coli strains (EHEC)
}

\author{
M. J. Rodrigues-Jesus ${ }^{1} \cdot$ W. L. Fotoran ${ }^{2} \cdot$ R. M. Cardoso $^{3} \cdot$ K. Araki $^{3} \cdot$ G. Wunderlich ${ }^{2} \cdot$ Luís C. S. Ferreira $^{1}$
}

Received: 4 July 2018 / Accepted: 24 September 2018 / Published online: 6 December 2018

(C) Sociedade Brasileira de Microbiologia 2018

\begin{abstract}
Microlipid vesicles (MLV) have a broad spectrum of applications for the delivery of molecules, ranging from chemical compounds to proteins, in both in vitro and in vivo conditions. In the present study, we developed a new set of nanosize multilayer lipid vesicles (NMVs) containing a unique combination of lipids. The NMVs enable the adsorption of histidine-tagged proteins at the vesicle surface and were demonstrated to be suitable for the in vivo delivery of antigens. The NMVs contained a combination of neutral (DOPC) and anionic (DPPG) lipids in the inner membrane and an external layer composed of DOPC, cholesterol, and a nickel-containing lipid (DGS-NTA [Ni]). NMVs combined with a recombinant form of the B subunit of the Shiga toxin $(\mathrm{rSt} \mathrm{x} 2 \mathrm{~B})$ produced by certain enterohemorragic Escherichia coli (EHEC) strains enhanced the immunogenicity of the antigen after parenteral administration to mice. Mice immunized with rStx2B-loaded NMVs elicited serum antibodies capable of neutralizing the toxic activities of the native toxin; this result was demonstrated both in vitro and in vivo. Taken together, these results demonstrated that the proposed NMVs represent an alternative for the delivery of antigens, including recombinant proteins, generated in different expression systems.
\end{abstract}

Keywords Nanoparticles $\cdot$ Delivery system $\cdot$ Multilamellar vesicles $\cdot$ Lipids vesicles $\cdot$ Shiga toxin

\section{Introduction}

During the last four decades, micro and nanosize particles of different chemical compositions have been intensively studied and successfully applied in several biomedical and biotechnological fields [1]. The possibility of targeting antigens to

Responsible Editor: Roxane Piazza

Electronic supplementary material The online version of this article (https://doi.org/10.1007/s42770-018-0035-0) contains supplementary material, which is available to authorized users.

Luís C. S. Ferreira

lcsf@usp.br

1 Vaccine Development Laboratory, Department of Microbiology, Institute of Biomedical Sciences, University of São Paulo, Av. Prof. Lineu Prestes, 1374, Cidade Universitária, São Paulo, SP 05508-900, Brazil

2 Unit for Drug Development and Plasmodium Molecular Biology, Department of Parasitology, Institute of Biomedical Sciences, University of São Paulo, São Paulo, Brazil

3 Supramolecular Chemistry and Nanotechnology Laboratory, Department of Fundamental Chemistry, Institute of Chemistry, University of São Paulo, São Paulo, Brazil specific cells of the immune system and the capacity to activate innate and/or adaptive immune responses by means of different types of vesicles are a relevant and promising platform for the development of vaccines and pharmaceutics formulations for clinical or veterinary use [2, 3]. Currently, several human vaccines are based on micro or nanoparticles, including those designed for the control of papillomaviruses and hepatitis B virus [2, 4]. Additionally, there are several ongoing clinical trials of formulations based on nano/microparticles targeting different infectious and chronic diseases [5].

Lipid-based particles, such as liposomes or lipid vesicles, are monolayer vesicles known for their ability to deliver antigens and to promote the activation of immune responses [6-12]. Liposomes are usually non-toxic, biodegradable, and capable of transporting soluble antigens in their hydrophilic or hydrophobic phases [13]. The immunological activity of liposome-based vaccine formulations relies on such factors as lipid composition, surface structure, size, lipid layer structure, preparation method, nature of the loaded antigen, and incorporation of immunomodulatory molecules, such as monophosphoryl lipid A (MPL-A). Such features may impact both the protection and release of the antigen, as well as the interaction with 
antigen presenting cells (APCs), leading to the modulation of mammalian species immune systems [2, 3, 14, 15].

Multilamellar lipid vesicles (MLV) are generated after fusion of vesicles in the presence of calcium or magnesium ions [16]. MLVs allow efficient encapsulation and a gradual and continuous release of antigens, which leads to enhanced antigen-specific immune responses [17-21]. These characteristics favor the application of MLVs, either alone or in combination with different adjuvants, of poorly immunogenic antigens, including several recombinant proteins, to promote the induction of humoral and/or cellular responses [9, 20, 22-25].

In this study, we report the use of nanosized MVLs (NMVs) employing a unique and innovative combination of lipids that enabled the incorporation of histidine-tagged antigens in both the intra-vesicle compartments and on the NMV surface. A recombinant antigen, specifically the B subunit of the type 2 Shiga toxin (Stx2B) produced by a number of enterohemorragic Escherichia coli (EHEC) strains, was employed as model to probe the immunogenicity of the NMV formulations. Stx2B is able to promote serious renal damage, including hemolytic uremic syndrome, within infected subjects $[26,27]$. However, since the Stx2B antigen is a weakly immunogenic agent, it requires the use of strong adjuvants or a suitable delivery system to induce large antibody responses capable of neutralizing the native Stx2B under experimental conditions [26, 28-35]. In this study, we addressed whether the encapsulation of rStx $2 \mathrm{~B}$ enhanced the immune response against this toxin in a mouse model.

\section{Methodology}

\section{Mice}

Female Balb/c mice, aged 6 to 8 weeks, were supplied to and maintained at the Department of Parasitology of the Institute for Biomedical Sciences at the University of São Paulo. Animal handling was performed according to the protocols for the use of animals in experimentation (license number 113 of the Ethics Committee on Animal Use of the University of São Paulo). Immunization was performed subcutaneously on the flanks of the animals, whereas serum samples were collected by submandibular bleeding. Mice were euthanized in $\mathrm{CO}_{2}$ chambers.

\section{Preparation of lipid nanosize multillamelar vesicles-NMVs}

NMVs were prepared with DOPC (1,2-dioleoyl-sn-glycero-3phosphocholine - catalog number 850375P), DPPG (1,2dipalmitoyl-sn-glycero-3-phospho-(1'-rac-glycerol)—catalog number 840455P) and 18:1 DGS-NTA (Ni) (1,2-dioleoyl-snglycero-3-[(N-(5-amino-1-carboxypentyl) iminodiacetic acid) succinyl] (nickel salt)—catalog number 790404P), and Cholesterol (catalog number 700000P) (all from AVANTI POLAR LIPIDS, Alabaster, AL). The internal vesicles used for protein incorporation were prepared with $1.26 \mu \mathrm{M}$ of the DOPC and DPPG phospholipids in a molar ratio of 3:1, corresponding to $970 \mu \mathrm{g}$ of lipid equivalent. The lipids were dissolved in chloroform and dried under nitrogen flow. The lipid film was suspended with $500 \mu \mathrm{g}$ OVA or rStx2B, which was previously prepared in phosphate buffer, and the mixture was incubated for $2 \mathrm{~h}$ at $37^{\circ} \mathrm{C}$ at $400 \mathrm{rpm}$ in an orbital shaker. Next, the emulsion was sonicated for 2 min at $240 \mathrm{~W}$ with the aid of a microtip to obtain a translucent material (BRANSON SONIFIER). After sonication, $\mathrm{CaCl}_{2}$ was added to a final concentration of $10 \mathrm{mM}$, and the samples were incubated for $2 \mathrm{~h}\left(37^{\circ} \mathrm{C}\right.$ at $\left.400 \mathrm{rpm}\right)$ for MLV formation [36]. The amount of unincorporated proteins was measured in aliquots centrifuged at $10,600 \times g$ for $5 \mathrm{~min}$. Proteins were quantified by a colorimetric method (BCA Kit-THERMO SCIENTIFIC). The outer vesicle preparation included $3.164 \mu \mathrm{M}$ of DOPC and cholesterol at a molar ratio of $4: 1$, corresponding to $2.24 \mathrm{mg}$ of lipid equivalent. The phospholipid DGS-NTA (Ni) used in the preparation of the outer vesicles corresponded to $15 \%$ of the total mass of DOPC:Cholesterol. The lipids were solubilized in chloroform and dried under a nitrogen flow forming a film, the film was later suspended in $10 \mathrm{mM}$ Bistrispropane buffer with $\mathrm{pH} 7.4$ (SIGMA) and agitated for $2 \mathrm{~h}$ at $37^{\circ} \mathrm{C}$ in an orbital shaker. The lipid emulsion was cleared by sonic treatment, as described above in the preparation of the internal vesicles. $\mathrm{NiCl}_{2}$ was added to a final concentration of $10 \mathrm{mM}$ followed by a new stirring step of $2 \mathrm{~h}$. The suspension containing the DOPC:Cholesterol:DGS-NTA (Ni) vesicles was centrifuged at $10,600 \times g$ for $5 \mathrm{~min}$, and the supernatant containing the smaller vesicles was transferred to the tube containing the internal vesicles (DOPC:DPPG) already coupled with the protein. The mixture was incubated at $400 \mathrm{rpm}$ for $18 \mathrm{~h}$ at $37^{\circ} \mathrm{C}$. The samples containing the NMVs were centrifuged at $10,600 \times g$ for $5 \mathrm{~min}$, and the pellet containing the NMVs was suspended in $300 \mu \mathrm{l}$ of phosphate buffered saline (PBS-pH 7.4) and stored at $4{ }^{\circ} \mathrm{C}$ until use. The amount of unincorporated protein was measured in the supernatant by colorimetric method (BCA Kit-THERMO SCIENTIFIC).

\section{Cryo transmission electron microscopy (Cryo-TEM)}

Liposomes were prepared as described above and analyzed by cryoelectron microscopy as previously published [37]. For this, $3 \mu \mathrm{l}$ protein-free liposomes were spotted to a holey carbon-film grid (Quantifoil Micro Tools GmbH, Jena, Germany), previously pretreated with Gatan Solarus 950 plasma cleaner. Specimen-coated grids were plunge-frozen in liquid ethane using a Gatan Cryoplunge 3 (Gatan Inc., Pleasanton, CA, USA). Low-dose imaging of the frozen, 
hydrated specimen kept in liquid nitrogen with a Gatan 626 single tilt cryoholder was performed on a JEM2100 electron microscope (JEOL Ltd., Tokyo, Japan, operating at $200 \mathrm{kV}$ ). A Gatan Ultrascan 4000 CCD camera was used to record images $(\sim \times 40,000$ magnification $)$.

\section{Measurement of the size and zeta potential of NMVs}

The NMV diameter and the polydispersity indices were determined by dynamic light scattering in the ZetaSizer Nano ZS equipment (MALVERN INSTRUMENTS). Aliquots (100 $\mu \mathrm{l})$ of the samples containing the NMV-OVA or NMV-rStx2B, as well as non-coupled NMVs, were suspended in $1 \mathrm{ml}$ of phosphate buffered saline (PBS). The polydispersity index of 0.0 corresponds to a sample containing homogeneous particles with a completely uniform size, whereas 1.0 indicates a sample with a distribution of broad particles sizes. The zeta potentials of the NMVs preparations were measured at room temperature in $1 \mathrm{ml}$ of sample volume. Samples with zeta potential larger than 30 , positive, or negative are generally considered to be stable colloidal suspensions.

\section{Immunization of mice with antigen-loaded NMVs}

$\mathrm{Balb} / \mathrm{c}$ mice were immunized with three subcutaneous (s.c.) doses (at an interval of 29 days), containing NMVs with either $25 \mu \mathrm{g}$ of $\mathrm{rStx} 2 \mathrm{~B}$ or OVA or equal concentrations of purified proteins only. A control immunization group received the protein-free NMVs. To evaluate the longevity of the induced antibody responses, serum samples were collected at 60,90 , and 105 days after the last dose by submandibular plexus bleeding. Another group of animals was submitted to a different immunization regimen with the same number of doses but with a 2-week interval between doses, to evaluate the influence of the interval between doses on the induction of antigenspecific humoral immune responses.

\section{Induction of antigen-specific serum antibody responses in mice immunized with NMVs}

Individual mouse serum samples were analyzed to determine the antigen-specific IgG, IgG1, and IgG2a titers. Enzymedlinked immunosorbent assays (ELISA) were performed in 96 well plates (COSTAR-CORNING-HIGH BINDING) coated with $100 \mu \mathrm{l}(1 \mu \mathrm{g} / \mathrm{ml})$ of the antigen diluted in PBS (pH 7.4) and kept at $4{ }^{\circ} \mathrm{C}$ for $18 \mathrm{~h}$ before use. Blocking was performed with $3 \%$ gelatin (SIGMA) in PBS for $2 \mathrm{~h}$ at $37^{\circ} \mathrm{C}$ (200 $\mu \mathrm{l}$ per well), followed by three washes with PBS containing $0.05 \%$ Tween 20 (PBS-T). Serum samples were diluted in $1 \%$ gelatin in PBS-T, applied to plate wells, submitted to twofold serial dilutions with the same buffer and kept at $37^{\circ} \mathrm{C}$ for $2 \mathrm{~h}$. The wells were washed with PBS-T and subsequently incubated for $2 \mathrm{~h}$ at $37^{\circ} \mathrm{C}$ with HRP-conjugated anti-mouse polyclonal IgG (SIGMA — catalog number A9044). In parallel, plaques were incubated with HRP-conjugated anti-mouse monoclonal IgG1 or IgG2a (SOUTHERN BIOTECHcatalog number 1070-05 and 1080-05) under the same conditions. After additional washes with PBS-T, the reactions were developed with o-phenylenediamine dihydrochloride substrate (OPD, SIGMA) and read at $492 \mathrm{~nm}$ on a plate reader (EPOCH model, BIOTEK).

\section{In vitro neutralizing effects of anti-rStx2B antibodies}

The in vitro Stx2 neutralizing antibody titers were determined by VERO cells assay $\left(2 \times 10^{4}\right.$ cells per well). The amount of tested Stx2a (purchased from Tufts University School of Medicine, Boston, MA, USA) was initially determined with cells exposed to different amounts of the toxin and cultured in $5 \% \mathrm{CO}_{2}$ at $37{ }^{\circ} \mathrm{C}$ for $48 \mathrm{~h}$. An aliquot of Stx2a $\left(1 \mathrm{CD}_{50}\right)$ capable of killing $50 \%$ of the cells $(15 \mathrm{ng} / \mathrm{ml}$ in a final volume $50 \mu \mathrm{l}$ ) was mixed with twofold serially diluted sera for $1 \mathrm{~h}$ at $37^{\circ} \mathrm{C}$ and $1 \mathrm{~h}$ at $4{ }^{\circ} \mathrm{C}$. The mixtures were transferred to microplates, with each well containing $2 \times 10^{4}$ VERO cells and incubated for 2 days in $5 \% \mathrm{CO}_{2}$ at $37{ }^{\circ} \mathrm{C}$. The control cells were incubated with a non-immune mouse serum sample and without addition of the toxin Stx2a. After washing with PBS, $100 \mu \mathrm{l}$ of crystal violet was added per well, and the plates were incubated at $37{ }^{\circ} \mathrm{C}$ for $15 \mathrm{~min}$. Additional washing was performed with distilled water, followed by incubation at room temperature for $20 \mathrm{~min}$ after addition of $100 \mu \mathrm{l}$ of acetic acid (99\%). Absorption readings were performed at $570 \mathrm{~nm}$ on the microplate reader. The percentage of viable cells was defined according to the formula $\left[\left(\mathrm{Abs}_{\text {toxin }+ \text { antibody }}-\mathrm{Abs}_{\text {toxin }}\right) /\right.$ $\left(\mathrm{Abs}_{\mathrm{no}}\right.$ toxin $\left.\left.-\mathrm{Abs}_{\mathrm{toxin}}\right)\right] \times 100$. The half maximal inhibitory concentrations $\left(\mathrm{IC}_{50}\right)$ of the anti-rStx $2 \mathrm{~b}$ antibody titers were calculated by non-linear regression curves using the Prism 6 program (GraphPad software, La Jolla, CA).

\section{In vivo anti-Stx2 serum neutralizing activity}

In vivo protective immunity to Stx 2 was determined in female Balb/c mice ( $n=10$ animals per group), immunized with different vaccine formulations. Mice were challenged by intravenously inoculated with $1 \mathrm{DL}_{100}$ corresponding to $3 \mathrm{ng}$ of Stx2a (120 ng/ $\mathrm{kg})$ and monitored daily for a period of 7 days.

\section{Statistical analyses}

Statistical analyses were performed with Prism 6 (Graph Pad software, La Jolla, CA). Data were analyzed using One or Two-way ANOVA for analyses of variance, followed by post-Bonferroni or $t$ test for multiple comparisons. $p$ values below 0.05 were considered to be statistically significant. All values are reported with mean \pm s.e.m. 


\section{Results}

\section{Preparation and physico-chemical analyses of antigen-loaded NMVs}

NMVs were loaded with OVA, as a model antigen, or rSx2B to evaluate the protective immunity associated with the antigen-specific antibodies. Based on the composition of the NMVs, the antigens are expected to remain adsorbed onto the vesicle outer surface and embedded in the hydrophilic portions of the lipid layers (Fig. 1a). Under cryo transmission electron microscopy, the NMVs formed bilamellar vesicles (Fig. 1b). Incorporation of OVA in the final NMV preparation corresponded on average to $55 \%$ of the total added protein, and the final preparation contained $74 \mu \mathrm{g}$ of protein $/ \mathrm{mg}$ of lipid. Regarding the distribution of the protein in the outer and inner vesicles, approximately $20 \%$ of the protein remained associated with the outer surface, while $80 \%$ was associated with the inner part of the vesicles (Table 1). NMVs were evaluated for antigen release rate, net electric charge, particle size, and polydispersity. Tests performed with NMVs loaded with OVA showed that approximately $50 \%$ of the protein was released during the first day of incubation at $37^{\circ} \mathrm{C}$, whereas most of the protein was released after a week (Fig. 1c). Similar protein release rates were observed during incubation of the NMV-OVA at $25^{\circ} \mathrm{C}$, while a lower release rate was observed at $4{ }^{\circ} \mathrm{C}$ (Fig. 1c). The NMV-OVA showed a negative residual surface electric charge of $-36.3 \mathrm{mV}$, while unloaded NMV presented a significantly lower surface potential of $-16.7 \mathrm{mV}$ (Table 1); this finding demonstrates the effect of OVA incorporation on the colloidal stability. Approximately $90 \%$ of the protein-free NMVs had an estimated size of $142.2 \mathrm{~nm}$, while NMV-OVA had an average size of $115.5 \mathrm{~nm}$ (Table 1). Both protein-free and NMV-OVA had polydispersion indices of 1 , indicating a broad size distribution but retaining the bilamellar structure.

Incorporation of $\mathrm{rStx} 2 \mathrm{~B}$ in the final NMV preparation corresponded to $80 \%$ of the total added protein, and the final preparation contained $109 \mu \mathrm{g}$ of protein $/ \mathrm{mg}$ of lipid, considering both the adsorption on the surface $(26 \%)$ and the distribution of the protein in the inner hydrophilic parts $(74 \%)$ of the vesicles, as shown in Table 1. Half of the incorporated protein was released after 3 days, irrespective of the incubation temperature (Fig. 1c). One week after incubation at either at $37{ }^{\circ} \mathrm{C}$ or $25{ }^{\circ} \mathrm{C}$, approximately $85 \%$ of the protein was released from the vesicles (Fig. 1c). NMV-rStx2B is constituted by approximately $115 \mathrm{~nm}$ diameter bilamellar vesicles with a net negative residual surface charge of $-14.4 \mathrm{mV}$ (Fig. S2), similar to that measured for NMV and significantly lower than that for NMV-OVA, and a polydispersity index of 0.79 (Table 1).
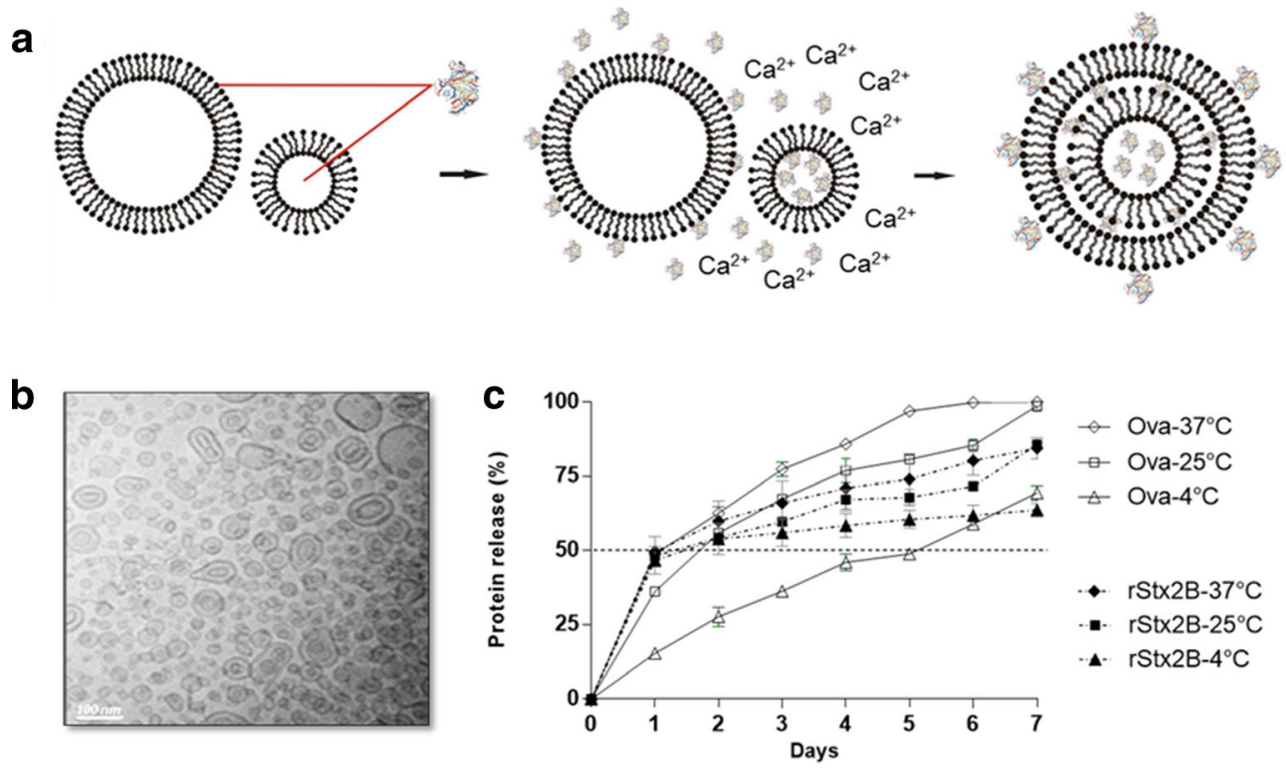

Fig. 1 Preparation and physical characterization of NMVs. a Schematic representation of the delivery system based on NMVs composed of anionic, neutral, and MPL-A phospholipids. The addition of $\mathrm{Ca}^{2+}$ ions promotes the entrance of the smaller vesicles into the larger ones generating stable fused bilamellar vesicles [38]. The protein is incorporated in the inner hydrophilic portions and/or is adsorbed on the surface. For example, the rStx2B protein is incorporated into the hydrophilic portions of the nanoparticle and is coupled to the outer membrane via the histidine tail (Stx2B structure reproduced from CONRADY, D.G. et al., 2010). b
Cryo transmission electron microscopy (Cryo-TEM) image of synthesized protein-free nanoparticles confirming the bilamellar structure of NMVs. The size distribution is quite broad with predominantly particles much smaller than $100 \mathrm{~nm}$ and few larger particles (scale bar $100 \mathrm{~nm}$ ). c Kinetics of protein release after incorporation in NMVs, considering the initial protein mass as $100 \%$, at 4,25 , and $37^{\circ} \mathrm{C}$ as a function of time in days. Values correspond to average \pm standard error of two experiments in duplicate 
Table 1 Characterization of the NMVs loaded with OVA or rStx2B

\begin{tabular}{llllll}
\hline Particles & $\begin{array}{l}\text { Incorporation } \\
\text { range }(\%)^{\mathrm{a}}\end{array}$ & $\begin{array}{l}\mathrm{O} / \mathrm{I} \text { ratio } \\
(\%)^{\mathrm{b}}\end{array}$ & $\begin{array}{l}\text { Zeta potential } \\
(\mathrm{mV})^{\mathrm{c}}\end{array}$ & ${\text { Size }(\mathrm{nm})^{\mathrm{d}}}$ & \begin{tabular}{l} 
Polidispersion index $_{(\mathrm{PDI})^{\mathrm{e}}}$ \\
\hline NMVs
\end{tabular} \\
NMV-Ova & $51.6-57.3(55)$ & 0.25 & $-16.7 \pm 4.75$ & $142.2 \pm 28.63$ & 0.05 \\
NMV-rStx2B & $57.8-91.4(80)$ & 0.35 & $-14.4 \pm 6.34$ & $115.0 \pm 25.90$ & 0.05 \\
\hline
\end{tabular}

(a) Percentage of OVA and rStx2B incorporation in NMVs. ${ }^{\text {(b) }}$ Distribution of proteins associated with the outer (O) and inner (I) vesicles. Values represent the percentage of protein coupled to the lipid vesicle relative to the total amount of protein added during the preparation of the MNVs. ${ }^{(c)}$ Zeta potential of the vesicles with and without the incorporated antigens (mean $+\mathrm{SD})$. ${ }^{\text {(d) }}$ Average diameters of NMVs (mean $\left.\pm \mathrm{SD}\right)$. ${ }^{\text {(e) }}$ Polydispersion Index (PDI) determined by dynamic light scattering (DLS) values main population of particles (square of the ratio between standard deviation and mean diameter of replicates). Values indicated in ${ }^{\mathrm{c}, \mathrm{d}}{ }^{\mathrm{a}}$ and ${ }^{\mathrm{e}}$ refer to mean $\pm \mathrm{SD}$ of three replicates with 13 readings each

\section{NMV as delivery vectors for induction of antigen-specific antibody responses}

Mice were immunized with NMV-OVA following an immunization regimen of three doses delivered subcutaneously at 29-day intervals (Fig. 2a). OVA-specific serum IgG titers reached maximal values 2 weeks after the third dose both in mice immunized with OVA and with NMV-OVA. NMV-
OVA-immunized mice produced an amount of OVA-specific serum IgG titers approximately two orders of magnitude larger than those immunized only with the protein (titer of $10^{6}$ in mice immunized with NMV-OVAs versus approximately $10^{4}$ in mice immunized with OVA protein 2 weeks after inoculation of the third dose) (Fig. 2b). The OVA-specific IgG titers declined continuously 60 and 90 days after the end of the immunization regimen, both in mice immunized with OVA

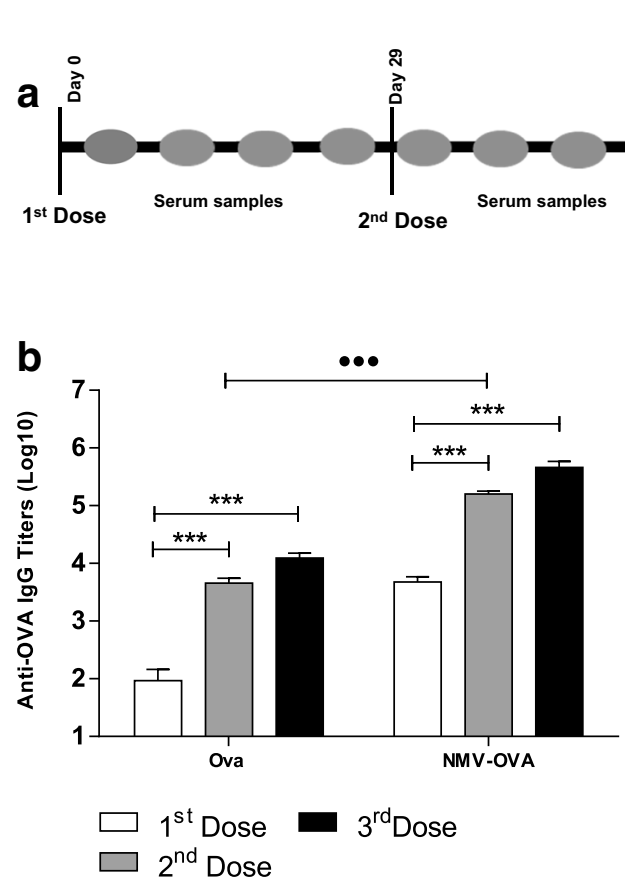

Fig. 2 NMV-OVA induce high antigen-specific titers and long-lasting serum antibody response. Female BALB/c mice ( $n=5 /$ group) were subcutaneously immunized with three doses of the vaccine formulations: OVA protein, NMV-OVA, and NMVs. Serum was collected on days $7,14,21$, and 28 after each dose and at 60,90 , and 105 days after the last dose. On day 91 after the last dose, mice were inoculated with a boost of the OVA antigen $(25 \mu \mathrm{g})$, except for the control group (NMV). a Schematic representation of the vaccination protocol. $\mathbf{b}$, c Anti-OVA-specific IgG antibody titers from sera of 14 days after each

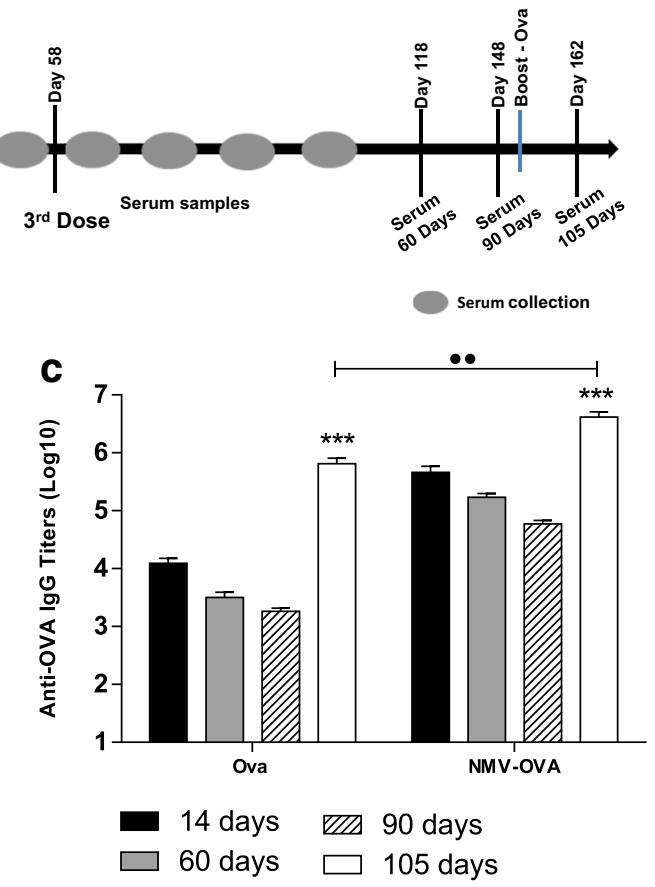

dose analyzed by ELISA (b) or 60, 90, and 105 days after the third dose (c). The antibody titers were determined by ELISA and the data correspond to mean values $\pm \mathrm{SD}$ of individual experiments carried out in duplicate. Titers values (MEAN $+2 \times \mathrm{SD}$ ) of the values obtained in the control group (NMV) were subtracted from the titer values determined for the other immunization groups. Statistical differences were determined by One-way ANOVA with Bonferroni post-test $* * * p<0.001$. Intergroup analysis carried out with Student's $t$ test was applied with $\cdots p<0.001$ and $\bullet p p<0.01$ 
and those immunized with OVA-MNVs. An additional dose of purified OVA $(25 \mu \mathrm{g})$, given subcutaneously to mice vaccinated with OVA or NMV-OVA, boosted the serum antibody responses. Notably, mice immunized with NMV-OVA elicited a considerably stronger response and considerably higher antiOVA IgG titers than in mice immunized with OVA (Fig. 2c). These results demonstrated that the incorporation of antigens into NMVs led to a significant increase in the antibody responses elicited in immunized mice.

\section{NMV-rStx2B induces high and long-lasting antibody responses capable of neutralizing Stx2}

Next, the vaccine potential of antigen-loaded NMVs was evaluated in mice. Protein rStx2B produced in E. coli cells retained its ability to bind to VERO cells and preserved the conformational epitopes that allow their recognition by antibodies raised against the native toxin (Fig. S1). NMV-rStx2B nanoparticles did not show any cytotoxic effects to mammalian cells (Fig. S3) but induced enhanced antigen-specific serum $\mathrm{IgG}$ responses after subcutaneous immunization in mice (Fig. 3a). NMV-rStx2B elicited maximal antibody titers 2 weeks after the end of the immunization regimen, and anti-rStx2B serum IgG titers were approximately twofold higher than those achieved in mice immunized only with the protein (Fig. 3b). The anti-rStx2B titers did not decrease significantly 2 or 3 months after the end of the immunization regimen when immunized with NMV-rStx2B nanoparticles. No increase in the antigen-specific antibody titers could be detected in immunized mice after a boost dose of purified rStx2B given 3 months after the end of the immunization regimen (Fig. 3c).

The properties of the anti-rStx2B antibodies produced in mice immunized with NMV-rStx2B were evaluated by determining their affinity to the respective antigen and their capacity to neutralize the native toxin. As shown in Fig. 3d, the antirStx2B showed enhanced antibody-antigen affinity after the boost dose with the purified protein 3 months after the last dose (Fig. 3d). We also measured the Stx2 neutralizing activity of antibodies raised in mice immunized with NMV-rStx2B using the Vero cell assay. Interestingly, the Stx2-neutralizing activity of sera collected from mice immunized with NMVrStx2B was significantly higher than those observed in mice immunized with the same amount of purified $r$ Stx2B protein (Fig. 3e). Notably, the boost with rStx2B, administered 3 months after the NMV-rStx2B immunization regimen, did not enhance the Stx2 neutralization titer, but the neutralizing effect remained superior compared with mice immunized only with rStx2B (Fig. 3e). These results demonstrate that the incorporation of $\mathrm{rStx} 2$ protein into NMVs enhanced the generation of antibodies capable of neutralizing the toxic effects of Stx2.

\section{In vivo Stx2-neutralizing activity of antibodies raised in mice immunized with NMV-rStx2B}

To demonstrate the protective immunity induced in mice immunized with NMV-rStx2B and to evaluate an alternative immunization regimen with a shorter interval between doses, a second immunization regimen was set. Mice were immunized with three vaccine doses with 2 -week intervals and 3 weeks after the last dose, the mice were challenged with a lethal dose of Stx2 (Fig. 4a). Based on this immunization regimen, mice immunized with NMV-rStx2B elicited antirStx2B serum IgG titers up to tenfold higher than those obtained upon immunization with rStx2B protein (Fig. 4b). Additionally, mice immunized with NMV-rStx2B produced a mixed Th1/Th2 response, as determined by the serum IgG subclass responses (Fig. 4c).

In order to determine the protective effects of anti-rStx2 antibodies, aliquots containing $15 \mathrm{ng} / \mathrm{ml}$ of Stx2a were incubated with sera collected 2 weeks after the last dose. Sera from mice immunized with NMV-rStx2B showed enhanced Stx2a neutralizing activity compared with those collected from mice immunized with purified rStx2B (Fig. 4d). Sera generated by administration of NMV-rStx2B and rStx2B showed a Stx2neutralization $\mathrm{IC}_{50}$ value of $365.7 \pm 46.3$ and $62.72 \pm 10.26$, respectively. Finally, $60 \%$ of the mice immunized with NMVrStx2B survived a lethal challenge with $1 \mathrm{LD}_{100}$ purified Stx2a, while none of those immunized with $\mathrm{rStx} 2 \mathrm{~B}$ or mice non-immunized survived after the third day under the tested experimental conditions (Fig.4e), demonstrating the significant enhancement of the neutralizing activity. These results demonstrate that the incorporation of rStx2B into NMVs enhanced the generation of antigen-specific antibodies and the protective immunity to Stx2a.

\section{Discussion}

A new class of NMVs was formed by fusion of two lipid vesicle preparations, an inner layer composed of neutral and anionic lipids (DOPC and DPPG) and an outer layer containing DOPC, cholesterol, and DGS-NTA (Ni) that enabled the coupling of recombinant His-tagged proteins as a platform for the delivery of antigens. The new lipid-based delivery system offers significant flexibility, enabling the incorporation of one or more antigens either adsorbed on the surface of the outer layers or embedded in lipid matrix. The system was also designed to enable the incorporation of adjuvant molecules, such as MPL-A, in order to further improve the activation of both innate and adaptive immune responses. Our results demonstrated that NMVs loaded with OVA or $\mathrm{rStx} 2 \mathrm{~B}$, produced in a recombinant expression system, enhanced the induction of antibody responses without measurable side effects in mice. The proposed nanosystem is a new and flexible platform for 


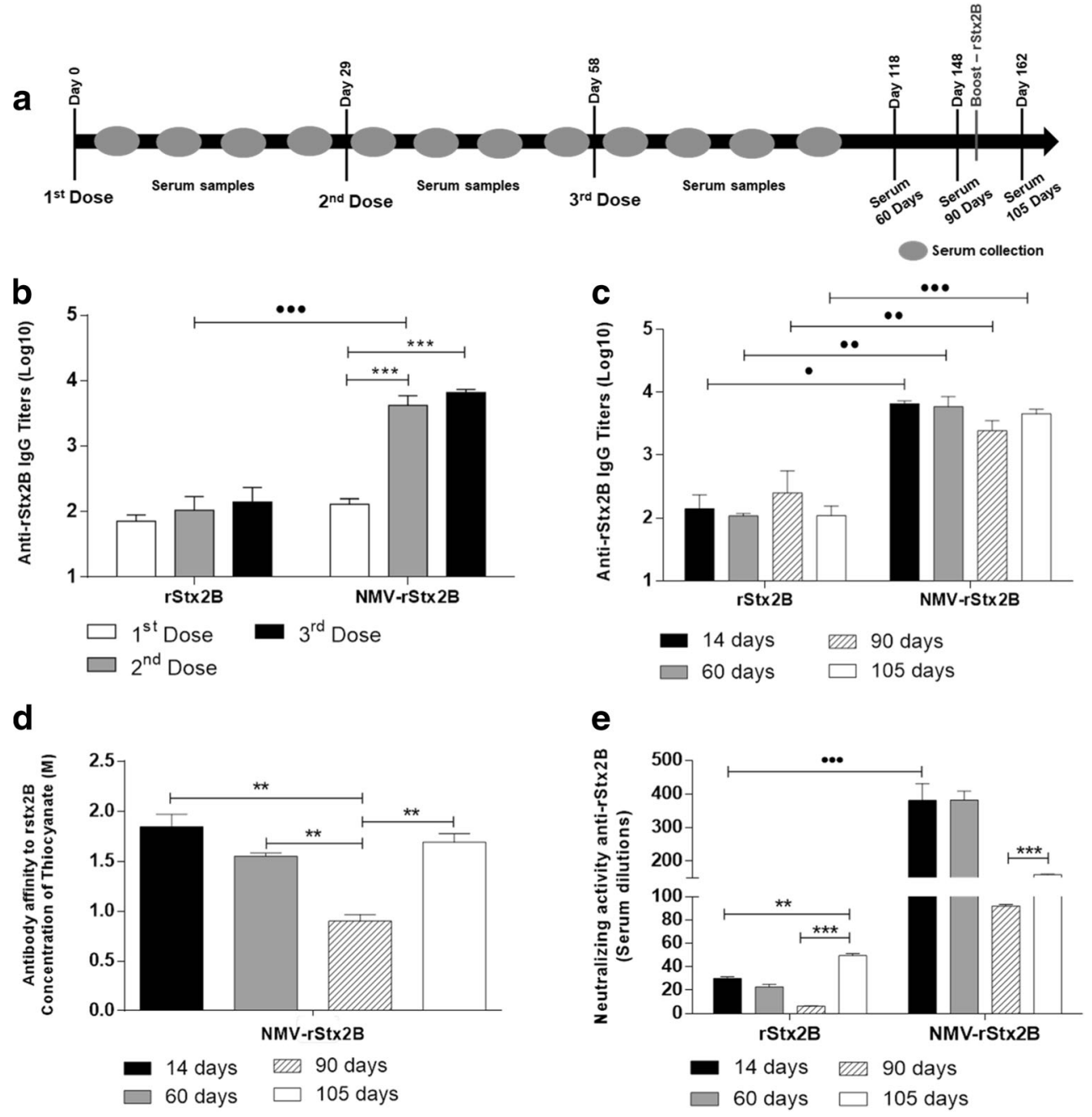

Fig. 3 NMV-rStx2B vaccine formulation improves magnitude and quality of the Stx2-specifi humoral immune response. Female BALB/c mice ( $n=5$ /group) were subcutaneously immunized with three doses of rStx2B or NMV-rStx2B. Ninety-one days after the last dose, the animals were inoculated with a $25-\mu \mathrm{g}$ dose of the $\mathrm{rStx} 2 \mathrm{~B}$ protein, except for the control group (NMV). Sera were collected on days 7, 14, 21, and 28 after each dose and at 60,90, and 105 days after the last dose and analyzed by ELISA assay (as described in the Methodology section), and the antirstx2 titers were measured. a Schematic representation of the vaccination protocol. $\mathbf{b}$, $\mathbf{c}$ Evaluation of anti-rStx $2 \mathrm{~B}$ IgG response from sera harvested 14 days after each vaccine dose (b) or 60, 90, and 105 days after the last dose (c). The antibody titers were determined by ELISA and the values obtained for the control group (NMV) were subtracted from the

the delivery of antigens, as confirmed by the enhanced induction of specific immune responses, with high potential to contribute to the development of more effective vaccines based on recombinant proteins.

The effectiveness of immune responses induced by the antigens delivered by NMV nanoparticles is directly proportional to the amount of antigen loading in both the inner and outer parts of the bilamellar vesicles. Under the tested preparation conditions, the incorporation of rStx2B in NMVs was demonstrated to be more efficient than of OVA $(80 \%$ and $55 \%$,

titers determined for the experimental groups (MEAN $+2 \times \mathrm{SD})$. $d$ Antibody affinity of the sera collected at different days after the third dose. Bars represent the molar concentrations of ammonium thiocyanate required to dissociate $50 \%$ of the antibodies bound to Stx2B. e Stx2 Neutralizing activity of the anti-Stx2B antibodies against the Stx2-induced cytotoxic effects in cell culture. The maximum dilution required to preserve $50 \%$ of the viable cells were determined for each serum sample. The data correspond to the mean values $\pm \mathrm{SE}$ of the individual values for each group in duplicate. Statistical differences are indicated by brackets: $* * * p<0.001, * * p<0.01, * p<0.05$, for one-way ANOVA with Bonferroni's post-test for the analysis as a function of the day each dose inoculation ${ }^{*} p<0.001, "{ }^{p} p<0.01, \bullet p<0.05$ ( $t$ test) for the intergroup analysis

respectively). Similarly, the amount of antigen accumulated in the outer lipid layer was higher for rStx2B than OVA; this finding was consistent with the fact that $\mathrm{rSt} 2 \mathrm{~B}$ was synthesized with a poly-histidine tail in contrast to OVA. In addition, it was demonstrated that a significant fraction of the OVA antigen associated with the outer layer does not require a histidine tag and thus does not depend on the interaction with the nickel coupled to DGS-NTA(Ni). The presence of protein molecules exposed on the NMVs surface increases the antigen loading and, more relevantly, contributes to the attraction and 

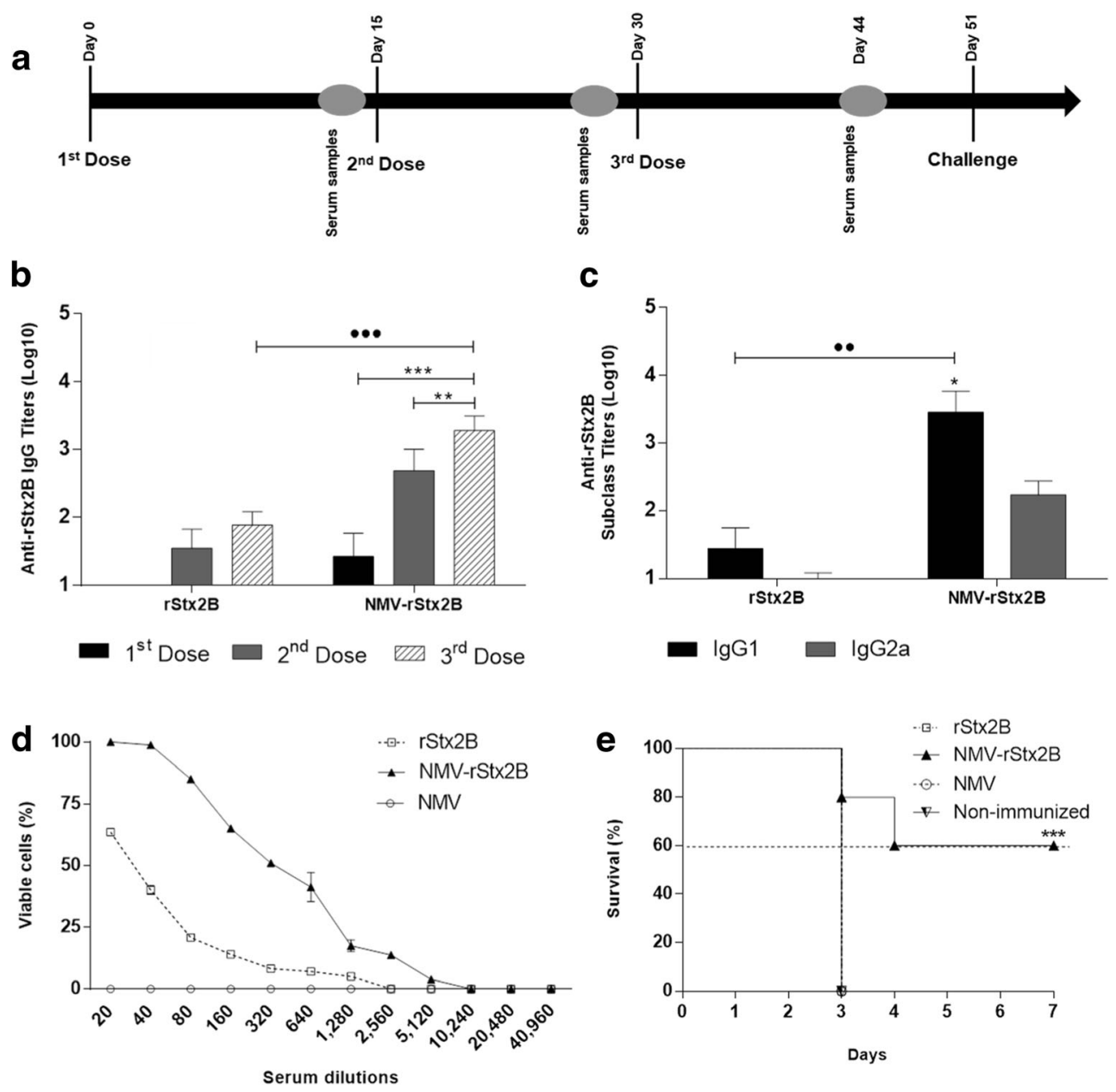

Fig. 4 Protective immunity to Stx 2 conferred by immunization with NMV-rStx2B. a Schematic representation of the tested vaccination regimen. BALB/c mice were immunized s.c. with three doses of each vaccine formulation: $r S t x 2 B$ or NMV-rStx2B. Blood samples were collected 14 days after the administration of each dose, and the challenge was carried out with Stx2a toxin 21 days after the last dose. b-c) Analysis of the serum Stx2B-specific total IgG and IgG subclass responses 14 days following each dose (b) or 14, 60, 90, and 105 days after the third dose $(\mathbf{c})$. The values represent the mean \pm SD $(n=10$ mice/group) for each group. Statistical differences were determined by one-way ANOVA with Bonferroni's post-hoc test in the analysis

considering the doses and the subclasses of each group $(* * * p<0.001$, $* p<0.05)$ and by $t$ test $(\cdots p<0.001, \bullet p<0.01)$ in the intergroup analysis. d In vitro neutralization of the Stx 2 toxicity. Vero cells were exposed to the recombinant Stx 2 holotoxin $\left(1 \times \mathrm{CD}_{50}-0.75 \mathrm{ng}\right)$ previously incubated with diluted tested serum samples. Values correspond to the average of two independent experiments performed in duplicates. e Protective immunity conferred by vaccination with NMV$\mathrm{rStx} 2 \mathrm{~B}$. Mice ( $n=10$ /group) were challenged with the toxin Stx $2(1 \times$ $\mathrm{LD}_{100}=3 \mathrm{ng}$ ) on the 21 st day after the last immunization dose. The nonimmunized group was not submitted to any treatment. Statistical analyses were carried out with the Mantel-Cox test $(* * * p<0.001)$

activation of antigen presenting cells (APCs), similar to other lipid-based delivery systems $[3,39]$. The effects of the NMVs depend on the combined action of both the lipid composition and the target protein antigen of the adjuvant delivery system. These characteristics enable the rational design and customization of the lipid vesicles for different vaccine strategies with a significant impact in the induced immune responses $[9,36$, 40, 41].

In addition to the relevant characteristics of vesicles and the antigen, the interval between doses was shown to have a significant impact on the levels of antibody production. When a 29-day interval between doses was used, no significant differences were observed in antigen-specific IgG titers from the second to third dose using NMV-OVA and NMV-rStx2B,

demonstrating that two doses would be sufficient to reach maximal antibody levels. The same profile was not observed when a 15-day dose regimen was used for immunization with NMV-rStx2B, which resulted in a significant difference between the titers achieved with the three dose regimen, which resulted in lower anti-rStx2B antibody titers. Shorter dose intervals may result in an inefficient induction of the immune responses against the antigen. Therefore, based on our observations, it is more appropriate to apply a longer dose interval with NMVs formulations. Most probably such formulations promote the continuous in vivo release of the antigen leading to an enhanced induction of immune responses.

The size of the NMVs is also a relevant feature in the activation of immune cells. Particles with sizes ranging from 
70 to $170 \mathrm{~nm}$ exhibited intermediate retention levels [42] and modulated the immune response in favor of activating antibody responses $[14,15]$. The fluidity of the lipid membranes is influenced by the transition temperature of the lipids used for the vesicle preparation $[2,9,24]$. We demonstrated that the protein release by NMVs also depends on the characteristics of the protein, but the release profile of the two tested antigens did not change with incubation temperature; the increase in temperature increased the membrane fluidity and the amount of proteins released. In contrast, the presence of the DGSNTA (Ni) phospholipid promoted their anchoring on the surface of vesicles via poly-histidine and Ni-NTA tails. Thus, coupling into NMVs preserves the antigenic integrity of the protein, which is a highly relevant feature to enhance the antigen-specific antibody responses $[18,43]$. Therefore, DGS-NTA (Ni) is a strong option for composing subunit vaccine delivery systems. In fact, the strategy of incorporating the antigen on the surface and in the interior of the NMVs was shown to be successful; it promotes its release at the inoculation site, as well as the continuous release from the inner parts, efficiently targeting and enhancing the serum IgG responses.

Shiga toxin (Stx) produced by EHEC strains is associated with the development of Hemolytic Uremic Syndrome (HUS), the most severe outcome associated with the infection by this pathogen. The non-toxic Stx B subunit has been a wellstudied antigen target for the development of vaccines capable of preventing HUS mortality and lifelong sequels $[26,33,34$, $38,44-48]$. According to a previous study, the protective effect to Stx is related to high levels of neutralizing antibodies and a long duration of the induced antibody response [35]. However, immunization with Stx B subunit requires the incorporation of strong adjuvants or employment of more effective delivery systems in order to overcome the naturally low immunogenicity of that protein $[34,35]$. In the present study, we demonstrated that the loading of rStx2B into NMVs (NMV-rStx2B) can significantly enhance the immunogenicity of the antigen in mice, increasing the levels of antibody to those achieved with the administration of ALUM the reference vaccine $[32,49]$. This finding can be ascribed to the presence of the antigen on the surface of the vesicles, which likely preserves conformational epitopes found in the native bacterial toxin, as well as the continuous release of the antigen from the interior, thereby enhancing the immune response. Interestingly, the amount of lipids in NMVs-Stx2B was significantly lower but induced stronger antibody responses than that reported in two previous studies based on unilamellar liposomes [50, 51].

\section{Conclusions}

Collectively, our results demonstrate that the NMV nanoparticles can be used as a convenient platform for incorporation of antigens and adjuvants to generate nanosystems exhibiting strong immunostimulatory effects that are adequate for the development of effective and safe vaccine formulations. Enhancement of the humoral immune responses against protein antigens by DOPC, DPPG and DGS-NTA (Ni) phospholipid multilamellar lipid nanovesicles was demonstrated. Significantly higher levels of antibodies were obtained upon administration of nanoparticle formulations loaded with OVA and $\mathrm{rSt} \times 2 \mathrm{~B}$ compared with the purified antigens. The protective effect of anti-rStx2B antibodies conferred partial but significant protection against the native Stx2a toxin, particularly when the mice were immunized according to a customized protocol. In summary, all results confirmed that NMVs carrying incorporated and surface-coupled protein antigens are efficient delivery systems for poorly immunogenic antigens, thereby exhibiting high potential for the development of subunit vaccine-based strategies.

Acknowledgments The authors would like to thank Eduardo Gimenez and Carolina Bertelli Ferreira for their technical support, Leticia Barbosa and Roxane Piazza of the Butantan Institute for the supply of the native Stx2a toxin used in this study, and Marina S. Palermo from the Faculty of Medicine at the University of Buenos Aires for supplying the pGEM-stx2 construction. All the data needed to evaluate the conclusions made in this paper are present within the data presented in the paper and/or the Supplemental Materials. Additional data may be requested from the authors.

Authors' contributions Jesus-Rodrigues, M.J. performed the rStx2B expression and purification assays, preparation of the NMVs, immunization and challenge of the mice, cell assays, antibody titers, data analysis, and wrote the manuscript. Fotoran, W.L. developed and assisted in the preparation of NMVs. Cardoso, R.M. carried out the physico-chemical characterization tests of NMVs. Araki, K. and Wunderlich, G. contributed scientific and technical assistance to the preparation and characterization of NMVs. Ferreira, L.C.S. supervised the study and the writing of the manuscript. All authors reviewed and commented on the manuscript.

Funding information This work was carried out at the Institute of Biomedical Sciences of the University of São Paulo and with financial support of the Research Support Foundation of the States of São Paulo (FAPESP_Process: 2014/21141-4 and 2015/17174-7).

\section{Compliance with ethical standards}

Conflict of interest The authors declare that they have no conflict of interest.

Publisher's Note Springer Nature remains neutral with regard to jurisdictional claims in published maps and institutional affiliations.

\section{References}

1. Allen TM, Cullis PR (2013) Liposomal drug delivery systems: from concept to clinical applications. Adv Drug Deliv Rev. Elsevier B.V.; 65(1):36-48. https://doi.org/10.1016/j.addr.2012. 09.037 
2. Watson DS, Endsley AN, Huang L (2012) Design considerations for liposomal vaccines: influence of formulation parameters on antibody and cell-mediated immune responses to liposome associated antigens. Vaccine. Elsevier Ltd; 30(13):2256-72. Available from: http://linkinghub.elsevier.com/retrieve/pii/S0264410X12001089

3. Smith DM, Simon JK, Baker JR (2013) Applications of nanotechnology for immunology. Nat Rev Immunol 13(8):592-605 Available from: http://www.nature.com/doifinder/10.1038/nri3488

4. Torres-Sangiao E, Holban AM, Gestal MC (2016) Advanced nanobiomaterials: vaccines, diagnosis and treatment of infectious diseases. Molecules 21(7):1-22

5. Yang L, Li W, Kirberger M, Liao W, Ren J (2016) Design of nanomaterial based systems for novel vaccine development. Biomater Sci. [cited 2018 Jan 18];4(5):785-802. Available from: http://xlink.rsc.org/?DOI=C5BM00507H

6. Afrin F, Anam K, Ali N (2000) Induction of partial protection against Leishmania donovani by promastigote antigens in negatively charged liposomes. J Parasitol 86(4):730-735 Available from: http://www.ncbi.nlm.nih.gov/pubmed/10958448

7. Migliaccio V, Santos FR, Ciancaglini P, Ramalho-Pinto FJ (2008) Use of proteoliposome as a vaccine against Trypanosoma cruzi in mice. Chem Phys Lipids 152(2):86-94

8. Malam Y, Loizidou M, Seifalian AM (2009) Liposomes and nanoparticles: nanosized vehicles for drug delivery in cancer. Trends Pharmacol Sci 30(11):592-599

9. Schmidt ST, Foged C, Korsholm KS, Rades T, Christensen D (2016) Liposome-based adjuvants for subunit vaccines: formulation strategies for subunit antigens and immunostimulators. Pharmaceutics 8(1):1-22

10. Fotoran WL, Colhone MC, Ciancaglini P, Stabeli RG, Wunderlich G (2016) Merozoite-protein loaded liposomes protect against challenge in two murine models of Plasmodium infection. ACS Biomater Sci Eng [Internet]. American Chemical Society; [cited 2018 Feb 25];2(12):2276-86. https://doi.org/10.1021/ acsbiomaterials.6b00492

11. Fotoran WL, Santangelo RM, Medeiros MM, Colhone M, Ciancaglini P, Barboza R, et al (2015) Liposomes loaded with P. falciparum merozoite-derived proteins are highly immunogenic and produce invasion-inhibiting and anti-toxin antibodies. J Control Release. Elsevier [cited 2018 Feb 25];217:121-7. Available from: https://www.sciencedirect.com/science/article/pii/ S0168365915300882?via\%3Dihub

12. Fotoran WL, Santangelo R, de Miranda BNM, Irvine DJ, Wunderlich G (2017) DNA-loaded cationic liposomes efficiently function as a vaccine against malarial proteins. Mol Ther Methods Clin Dev. American Society of Gene \& Cell Therapy; [cited 2018 Feb 25];7:1-10. Available from: http://www.ncbi.nlm.nih.gov/ pubmed/28879213

13. Torchilin VP (2005) Recent advances with liposomes as pharmaceutical carriers. Nat Rev Drug Discov 4(2):145-160

14. Brewer JM, Tetley L, Richmond J, Liew FY, Alexander J (1998) Lipid vesicle size determines the Th1 or Th2 response to entrapped antigen. J Immunol 161(8):4000-4007

15. Brewer JM, Pollock KGJ, Tetley L, Russell DG (2004) Vesicle size influences the trafficking, processing, and presentation of antigens in lipid vesicles. J Immunol 173(10):6143-6150

16. Papahadjopoulos D, Nir S, Düzgünes N (1990) Molecular mechanisms of calcium-induced membrane fusion. J Bioenerg Biomembr 22(2):157-179

17. DeMuth PC, Moon JJ, Suh H, Hammond PT, Irvine DJ (2012) Releasable layer-by-layer assembly of stabilized lipid nanocapsules on microneedles for enhanced transcutaneous vaccine delivery. ACS Nano. NIH Public Access; [cited 2016 Oct 13];6(9):804151. Available from: http://www.ncbi.nlm.nih.gov/pubmed/ 22920601
18. Pejawar-Gaddy S, Kovacs JM, Barouch DH, Chen B, Irvine DJ (2014) Design of lipid nanocapsule delivery vehicles for multivalent display of recombinant Env trimers in HIV vaccination. Bioconjug Chem. [cited 2016 Oct 13];25(8):1470-8. Available from: http://www.ncbi.nlm.nih.gov/pubmed/25020048

19. Foged C, Arigita C, Sundblad A, Jiskoot W, Storm G, Frokjaer S (2004) Interaction of dendritic cells with antigen-containing liposomes: effect of bilayer composition. Vaccine 22(15-16):19031913

20. Genito CJ, Beck Z, Phares TW, Kalle F, Limbach KJ, Stefaniak ME et al (2017) Liposomes containing monophosphoryl lipid A and QS-21 serve as an effective adjuvant for soluble circumsporozoite protein malaria vaccine FMP013. Vaccine 35(31):3865-3874. https://doi.org/10.1016/j.vaccine.2017.05.070

21. Joo K-I, Xiao L, Liu S, Liu Y, Lee C-L, Conti PS, et al (2013) Crosslinked multilamellar liposomes for controlled delivery of anticancer drugs. Biomaterials. [cited 2018 Feb 13];34(12):3098-109. Available from: https://www.ncbi.nlm.nih.gov/pmc/articles/ PMC3995748/pdf/nihms442471.pdf

22. Alinaghi A, Rouini MR, Johari Daha F, Moghimi HR (2014) The influence of lipid composition and surface charge on biodistribution of intact liposomes releasing from hydrogel-embedded vesicles. Int J Pharm. Elsevier B.V.; 459(1-2):30-9. https://doi.org/10.1016/j. ijpharm.2013.11.011

23. Luo Y, Liu Z, Zhang X, Huang J, Yu X, Li J et al (2016) Effect of a controlled-release drug delivery system made of oleanolic acid formulated into multivesicular liposomes on hepatocellular carcinoma in vitro and in vivo. Int $J$ Nanomedicine. [cited 2016 Oct 4];11: 3111-29. Available from: http://www.ncbi.nlm.nih.gov/pubmed/ 27471381

24. Giddam AK, Zaman M, Skwarczynski M, Toth I (2012) Liposomebased delivery system for vaccine candidates: constructing an effective formulation. Nanomedicine [Internet]. Future Medicine Ltd London, UK; [cited 2018 Jan 17];7(12):1877-93. Available from: http://www.futuremedicine.com/doi/10.2217/nnm.12.157

25. Mazumder S, Maji M, Ali N (2011) Potentiating effects of MPL on DSPC bearing cationic liposomes promote recombinant GP63 vaccine efficacy: high immunogenicity and protection. PLoS Negl Trop Dis 5(12)

26. Mukherjee J, Chios K, Fishwild D, Hudson D, O’Donnell S, Rich SM et al (2002) Human Stx2-specific monoclonal antibodies prevent systemic complications of Escherichia coli $\mathrm{O} 157: \mathrm{H} 7$ infection. Infect Immun. American Society for Microbiology (ASM); [cited 2016 Oct 19];70(2):612-9. Available from: http://www.ncbi.nlm. nih.gov/pubmed/11796590

27. Smith JL, Fratamico PM, Gunther NW (2014) Shiga toxinproducing Escherichia coli, 1st edn. Adv Appl Microbiol. Copyright (C) 2014 Elsevier Inc. All rights reserved, pp 145-197. https://doi.org/10.1016/B978-0-12-800262-9.00003-2

28. Luz D, Chen G, Maranhão AQ, Rocha LB, Sidhu S, Piazza RMF (2015) Development and characterization of recombinant antibody fragments that recognize and neutralize in vitro Stx2 toxin from Shiga toxin-producing Escherichia coli. PLoS One. [cited 2016 Oct 19];10(3):e0120481. Available from: http://www.ncbi.nlm. nih.gov/pubmed/25790467

29. Bernedo-Navarro RA, Miyachiro MM, Da Silva MJ, Reis CF, Conceição RA, Gatti MSV et al (2014) Peptides derived from phage display libraries as potential neutralizers of Shiga toxin-induced cytotoxicity in vitro and in vivo. J Appl Microbiol 116(5):13221333

30. Mejias MP, Hiriart Y, Lauche C, Fernandez-Brando RJ, Pardo R, Bruballa A et al (2016) Development of camelid single chain antibodies against Shiga toxin type 2 (Stx2) with therapeutic potential against Hemolytic uremic syndrome (HUS). Sci Rep. Nature Publishing Group; 6(April):24913. Available from: http://ovidsp. 
ovid.com/ovidweb.cgi? $\mathrm{T}=\mathrm{JS} \& \mathrm{PAGE}=$ reference $\& \mathrm{D}=$ prem $\& N E W S=N \& A N=27118524$

31. Bentancor LV, Bilen M, Brando RJF, Ramos MV, Ferreira LCS, Ghiringhelli PD et al (2009) A DNA vaccine encoding the enterohemorragic Escherichia coli Shiga-like toxin 2 A2 and B subunits confers protective immunity to Shiga toxin challenge in the murine model. Clin Vaccine Immunol 16(5):712-718

32. Mejias MP, Ghersi G, Craig PO, Panek CA, Bentancor LV, Baschkier A et al (2013) Immunization with a chimera consisting of the B subunit of Shiga toxin type 2 and Brucella lumazine synthase confers total protection against Shiga toxins in mice. $\mathrm{J}$ Immunol 191(5):2403-2411 Available from: http://www.ncbi. nlm.nih.gov/pubmed/23918978

33. Rojas RLG, Gomes PADP, Bentancor LV, Sbrogio-Almeida ME, Costa SOP, Massis LM et al (2010) Salmonella enterica serovar typhimurium vaccine strains expressing a nontoxic Shiga-like toxin 2 derivative induce partial protective immunity to the toxin expressed by enterohemorrhagic Escherichia coli. Clin Vaccine Immunol 17(4):529-536

34. Gomes PAD, Bentancor LV, Paccez JD, Sbrogio-Almeida ME, Palermo MS, RCC F et al (2009) Antibody responses elicited in mice immunized with Bacillus subtilis vaccine strains expressing Stx2B subunit of enterohaemorragic Escherichia coli O157:H7. Brazilian J Microbiol 40:333-338

35. Garcia-Angulo VA, Kalita A, Torres AG. Advances in the development of enterohemorrhagic Escherichia coli vaccines using murine models of infection

36. Reviakine I, Simon A, Brisson A (2000) Effect of $\mathrm{Ca}^{2+}$ on the morphology of mixed DPPC-DOPS supported phospholipid bilayers. Langmuir. [cited 2018 Feb?13];16(4):1473-7. Available from: http://pubs.acs.org/doi/abs/10.1021/la990806g

52. Casal HL, Martin A, Mantsch HH, Paltauf F, Hauser H (1987) Infrared studies of fully hydrated unsaturated phosphatidylserine bilayers. Effect of lithium and calcium. Biochemistry. [cited 2018 Feb?13];26(23):7395-401. Available from: http://pubs.acs.org/doi/ abs/10.1021/bi00397a030

37. Moon JJ, Suh H, Li AV, Ockenhouse CF, Yadava A, Irvine DJ (2012) Enhancing humoral responses to a malaria antigen with nanoparticle vaccines that expand $\mathrm{Tfh}$ cells and promote germinal center induction. Proc Natl Acad Sci U S A 109:1080-1085

38. Capozzo AVE, Creydt VP, Dran G, Ferna G, Bentancor LV, Rubel C et?al (2003) Development of DNA vaccines against hemolyticuremic syndrome in a murine model. Society 71(7):3971-3978

39. Schwendener RA (2014) Liposomes as vaccine delivery systems: a review of the recent advances. Ther Adv Vaccines 2(6):159-182 Available from: http://tav.sagepub.com/lookup/doi/10.1177/ 2051013614541440

40. Casal HL, Martin A, Mantsch HH, Paltauf F, Hauser H (1987) Infrared studies of fully hydrated unsaturated phosphatidylserine bilayers. Effect of lithium and calcium. Biochemistry. [cited 2018 Feb?13];26(23):7395-401. Available from: http://pubs.acs.org/doi/ abs/10.1021/bi00397a030

41. Tah B, Pal P, Mishra S, Talapatra GB (2014) Interaction of insulin with anionic phospholipid (DPPG) vesicles. Phys Chem Chem
Phys. The Royal Society of Chemistry; [cited 2018 Feb?13];16(39):21657-63. Available from: http://xlink.rsc.org/? $\mathrm{DOI}=\mathrm{C} 4 \mathrm{CP} 03028 \mathrm{~A}$

42. Oussoren C, Zuidema J, Crommelin DJA, Storm G (1997) Lymphatic uptake and biodistribution of liposomes after subcutaneous injection. II. Influence of liposomal size, lipid composition and lipid dose. Biochim Biophys Acta Biomembr 1328(2):261272

43. Patel JD, O'Carra R, Jones J, Woodward JG, Mumper RJ (2007) Preparation and characterization of nickel nanoparticles for binding to His-tag proteins and antigens. Pharm Res. Springer US; [cited 2018 Jan 16];24(2):343-52. Available from: http://link.springer. com/10.1007/s11095-006-9154-7

44. Smith MJ, Teel LD, Carvalho HM, Melton-Celsa AR, O'Brien AD (2006) Development of a hybrid Shiga holotoxoid vaccine to elicit heterologous protection against Shiga toxins types 1 and 2. Vaccine 24(19):4122-4129

45. Tsuji T, Shimizu T, Sasaki K, Tsukamoto K, Arimitsu H, Ochi S et al (2008) A nasal vaccine comprising B-subunit derivative of Shiga toxin 2 for cross-protection against Shiga toxin types 1 and 2. Vaccine 26(17):2092-2099

46. Arimitsu H, Sasaki K, Iba Y, Kurosawa Y, Shimizu T, Tsuji T (2015) Isolation of B subunit-specific monoclonal antibody clones that strongly neutralize the toxicity of Shiga toxin 2. Microbiol Immunol 59(2):71-81

47. Melton-Celsa AR (2014) Shiga toxin (Stx) classification, structure, and function. Microbiol Spectr. NIH Public Access; [cited 2018 Jan 17];2(4):EHEC - 0024-2013. Available from: http://www. ncbi.nlm.nih.gov/pubmed/25530917

48. Olavesen KK, Lindstedt B-A, Løbersli I, Brandal LT (2016) Expression of Shiga toxin 2 (Stx2) in highly virulent Stxproducing Escherichia coli (STEC) carrying different antiterminator (q) genes. Microb Pathog. Academic Press; [cited 2018 Jan 17];97:1-8. Available from: http://www.sciencedirect. com/science/article/pii/S088240101630105X?via\%3Dihub

49. Mejias MP, Cabrera G, Fernández-Brando RJ, Baschkier A, Ghersi G, Abrey-Recalde MJ et al (2014) Protection of mice against Shiga toxin 2 (Stx2)-associated damage by maternal immunization with a Brucella lumazine synthase-Stx2 B subunit chimera. Infect Immun 82(4):1491-1499

50. Fukuda T, Kimiya T, Takahashi M, Arakawa Y, Ami Y, Suzaki Y et al (1998) Induction of protection against oral infection with cytotoxin-producing Escherichia coli O157:H7 in mice by Shigalike toxin-liposome conjugate. Int Arch Allergy Immunol 116(4): 313-317 Available from: http://www.ncbi.nlm.nih.gov/entrez/ query. fcgi? $\mathrm{cmd}=$ Retrieve $\& \mathrm{db}=$ PubMed $\&$ dopt $=$ Citation $\&$ list uids $=9693282$

51. Suzaki Y, Ami Y, Nagata N, Naito S, Kato H, Taneichi M et al (2002) Protection of monkeys against Shiga toxin induced by Shiga toxin-liposome conjugates. Int Arch Allergy Immunol 127(4):294-298 Avrailable from: http://www.ncbi.nlm.nih.gov/ pubmed/12021548 